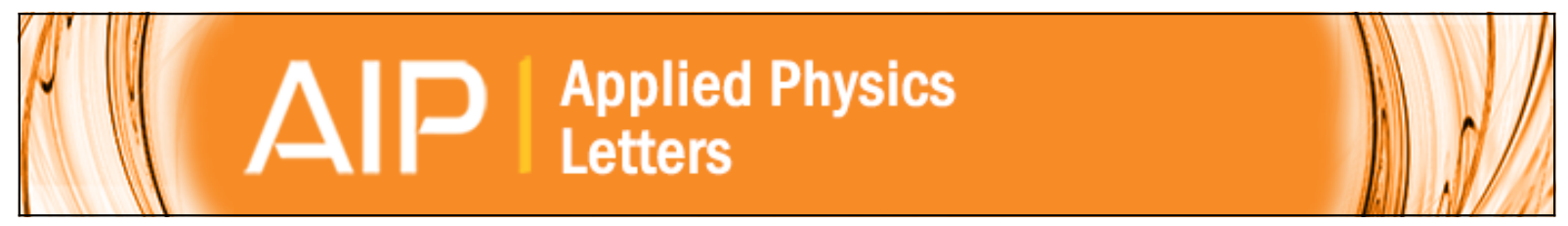

\title{
Self-heated fiber Bragg grating sensors
}

Kevin P. Chen, Ben McMillen, Michael Buric, Chuck Jewart, and Wei Xu

Citation: Applied Physics Letters 86, 143502 (2005); doi: 10.1063/1.1895485

View online: http://dx.doi.org/10.1063/1.1895485

View Table of Contents: http://scitation.aip.org/content/aip/journal/apl/86/14?ver=pdfcov

Published by the AIP Publishing

\section{Articles you may be interested in}

Pressure effects on the temperature sensitivity of fiber Bragg gratings

AIP Conf. Proc. 1511, 1570 (2013); 10.1063/1.4789229

Development of optical fiber Bragg grating force-reflection sensor system of medical application for safe minimally invasive robotic surgery

Rev. Sci. Instrum. 82, 074301 (2011); 10.1063/1.3606502

A multiplexed fiber Bragg grating sensor for simultaneous salinity and temperature measurement

J. Appl. Phys. 103, 053107 (2008); 10.1063/1.2890156

Fiber Bragg grating microphone system

Appl. Phys. Lett. 89, 161109 (2006); 10.1063/1.2363977

Highly sensitive fiber Bragg grating refractive index sensors

Appl. Phys. Lett. 86, 151122 (2005); 10.1063/1.1904716

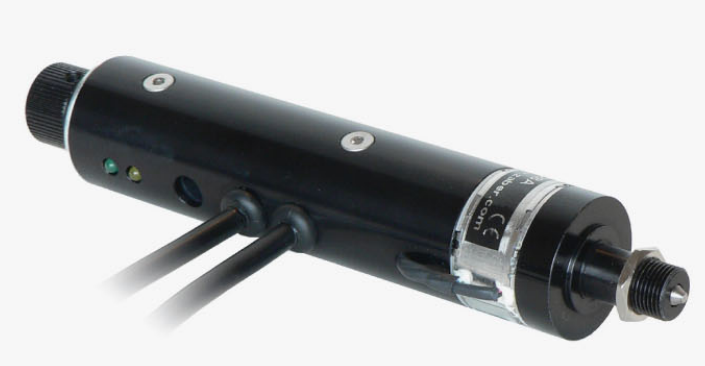

Automate your set-up with 


\title{
Self-heated fiber Bragg grating sensors
}

\author{
Kevin P. Chen, ${ }^{a)}$ Ben McMillen, Michael Buric, and Chuck Jewart \\ Department of Electrical and Computer Engineering, University of Pittsburgh, \\ Pittsburgh, Pennsylvania 15261
}

Wei Xu

StockerYale Inc., Salem, New Hampshire 03079

(Received 14 December 2004; accepted 10 February 2005; published online 28 March 2005)

\begin{abstract}
This letter demonstrates an approach for tuning fiber Bragg grating sensors with optical energy carried in the same optical fiber. Optical energy carried in the optical fiber was used to heat in-fiber Bragg gratings in order to alter the grating's optical response to surrounding media. The functional enhancement of optically heated Bragg gratings as sensor devices is demonstrated by a dual-function Bragg grating temperature and level sensing array for liquid at room and cryogenic temperatures. (C) 2005 American Institute of Physics. [DOI: 10.1063/1.1895485]
\end{abstract}

Fiber Bragg gratings (FBGs) are key components for optical sensing and communication. ${ }^{1}$ In-fiber sensing components offer several important advantages over other optical and electronic devices, including immunity to electromagnetic fields, long lifetimes, high sensitivity, and the capability of working in harsh environments. Despite the advantages of purely optical fiber components, their performance and applications have been limited by their total passivity. Passive sensor elements do not support active adjustment of sensing parameters to adapt to a changing environment. In order to enhance the functionality of fiber-optic components, intensive research has been carried out in the past few years directed toward the development of tunable FBGs. Tuning by various methods including on-fiber electrical heating, ${ }^{2-4}$ electrowetting, ${ }^{5}$ piezo-electric actuation, ${ }^{6}$ and mechanical actuation ${ }^{7}$ has been demonstrated. Despite advances in tunability, a common drawback for all active fiber components still remains. In order to tune a fiber device, an energy source, an energy delivery mechanism, and on-fiber actuators are required. To date, electricity has been the only form of energy used to power in-fiber devices. An electrical cable must run along with the optical fiber to supply this energy. Additional cabling, however, leads to many problems including an increase in manufacturing cost due to the additional on-fiber electrical contacts, the need for delicate packaging, and the elimination of electromagnetic immunity. Due to these issues, such fiber components would no longer be suitable for use in hostile environments. Improvements in the performance and functionality of an electrically driven active fiber component are achieved at the expense of many of the intrinsic advantages possessed by a purely optical component.

In this letter we demonstrate a concept that active FBG sensors can be directly powered by in-fiber light as illustrated in Fig. 1. In contrast to a passive sensor, optical power is delivered together with a sensing signal through the same fiber that contains FBGs. Optical taps are fabricated in desired sections of the optical fiber to release high-power laser light as an energy source. An optical conversion membrane coated on the outside of the optical fiber will serve as a

${ }^{a}$ Author to whom correspondence should be addressed; electronic mail: kchen@engr.pitt.edu transducer converting high-power laser energy to other energy forms. The optical characteristics of FBGs can then be adjusted using this optical energy. By eliminating the electric cabling, on-fiber electric contacts, and delicate packaging, the functional enhancement of FBG sensors can be achieved without compromising any intrinsic advantages of in-fiber passive sensors.

To demonstrate this concept, we present a dual-function active FBG sensor array for liquid temperature and level sensing at both room and cryogenic temperatures. Currently, the temperature and level of liquid hydrogen in cryogenic fuel tanks for space missions is detected with an array of point sensors using silicon diodes. During operation, the silicon diodes are heated by current pulses. The voltage drop across a diode- sensor is used to gauge the temperature rise of the heated diode. When the diode is submerged in cryogenic fuel, the temperature rise of the heated diode is limited by the boiling point of the liquid hydrogen. The temperature difference between the submerged diodes and diodes outside the liquid is utilized to determine the liquid level. Since liquid fuel does not settle to the bottom of the tank in a microgravity environment, a large number of diode point sensors are needed to precisely gauge the distribution and the total mass of the liquid fuel inside fuel tanks. Each point sensor requires two metal leads to measure the voltage and supply current to the sensor. This incurs a large number of electrical feedthrough lines for one cryogenic fuel tank. The large number of metal feedthrough lines increases heat leakage and the potential for mechanical and electrical failure in a high-g environment. The large number of electronic sensors

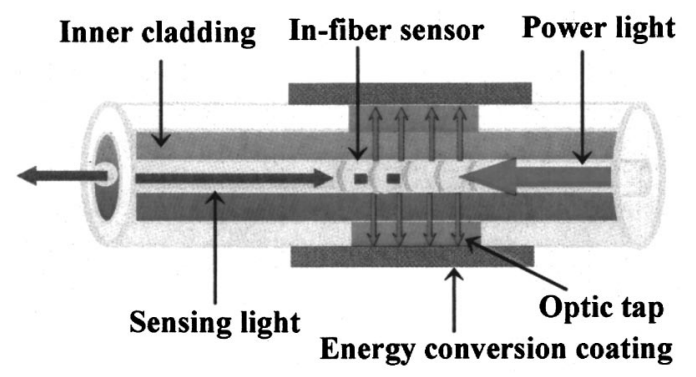

FIG. 1. Schematic illustration of active FBG which is heated by in-fiber high-power laser light. 


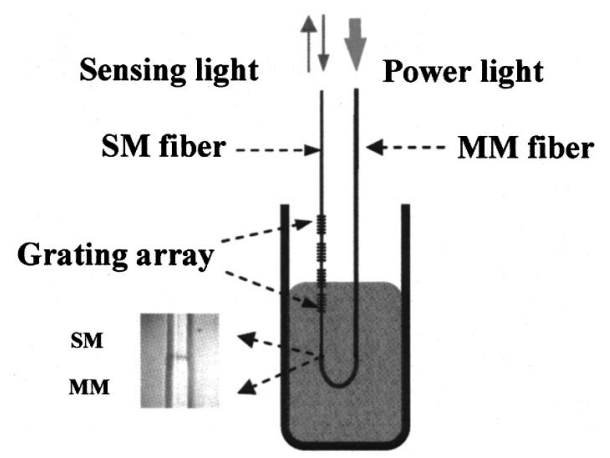

FIG. 2. Sketch of a grating level sensor array, light from a high-power laser diode was coupled into a multimode (MM) fiber that is fusion spliced to the SM fiber. High-power $910 \mathrm{~nm}$ laser light in the multimode fiber leaks into the cladding of the single-mode fiber through the spliced junction and heats the silver coating of FBGs. The actual fusion spliced SM to MM junction is shown in the figure.

and metal leads are also easily susceptible to electromagnetic interference, because any ac current imposed upon them is rectified and appears as an erroneous temperature offset.

This practical problem can be readily resolved by the active fiber sensing technology proposed in this letter. The dual-function level/temperature sensor demonstrated in this letter consists of a FBG array powered by in-fiber light. When the power light is turned off, the FBG can be used as a temperature sensor to precisely gauge the temperature distribution in the fuel tank. When the power light is turned on, optical energy carried by the fiber is used to heat fiber Bragg grating sensor arrays to distinguish between the liquid and gas phases. The spectral thermal response of grating sensors is detected and transmitted back through the same optical fiber. This work promises a true one-fiber solution for cryogenic level/temperature sensing without a single electric wire and with only one fiber feedthrough.

The self-heated FBG level sensor demonstrated herein involves two sections of fiber as shown in Fig. $2{ }^{8}$ A FBG was first written in a single-mode standard fiber. The reflection spectrum of the FBG was monitored with an optical spectrum analyzer and a broadband source via a circulator. The single-mode fiber was fusion spliced with a $100-\mu \mathrm{m}$ -diam core multimode fiber. Ten watts of $910 \mathrm{~nm}$ laser light from a high-power diode laser array was coupled into the free end of the multimode fiber using a pair of $20 \times$ microscope objectives. The $910 \mathrm{~nm}$ laser light propagating in the multimode fiber leaked into the cladding of the single-mode fiber through the spliced junction. The light leakage profile was measured using a cut-back technique. By measuring 910 $\mathrm{nm}$ laser light coupling out of the fiber as a function of distance from the splice junction, it was found that the light energy leaking out from the fiber cladding falls off exponentially with distance. Ninety percent of the light was lost within $10 \mathrm{~cm}$ of the splice junction. A uniform silver film was plated on the FBG section of the fiber to absorb light. The coated section began approximately $5 \mathrm{~mm}$ from the splice junction and had a resistance of $2.2 \Omega / \mathrm{cm}$. Uniform gratings with lengths of $10 \mathrm{~mm}$ were used in the experiments.

The liquid sensing experiments were carried out in both water and liquid nitrogen. Figure 3 shows a Bragg grating spectral response in both water and air. When the $910 \mathrm{~nm}$ power laser was turned off, the grating served as a tempera-

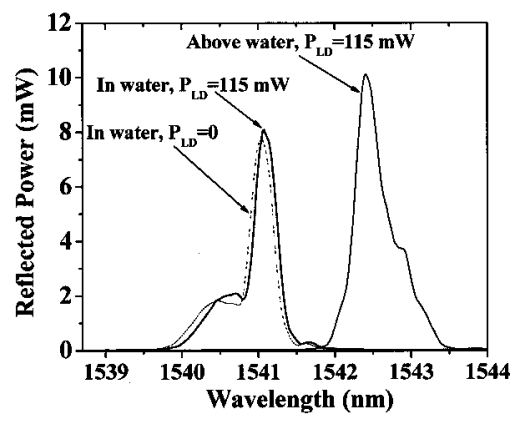

FIG. 3. Bragg grating response in and out of water when it was heated by $115 \mathrm{~mW} 910 \mathrm{~nm}$ diode laser power.

ture sensor. To determine whether the grating is submersed inside the liquid (level sensing), the $910 \mathrm{~nm}$ laser was turned on to inject $115 \mathrm{~mW}$ to heat the grating. When the grating is submersed under water, the resonance peak (solid trace) of the heated grating is shifted about $60 \mathrm{pm}$ from the unheated peak (dotted trace). When the grating was pulled above the water level, the grating reflection peak rapidly shifted over $1.4 \mathrm{~nm}$ from 1541 to $1542.4 \mathrm{~nm}$. The reflection spectrum of the FBG is also changed, probably due to the nonuniform silver coating on the fiber. However, the dramatic thermal response for the heated grating provided unambiguous detection of the liquid level. The distinct thermal responses of heated gratings have their origins in the disparate thermal properties of air and water. The thermal responses of heated gratings in air, water, and liquid nitrogen as a function of input laser power are further characterized by Fig. 4. As expected, the heated grating exposed to air produced the largest resonance wavelength shift. The peak shift of the heated grating in ambient room temperature air follows a linear variation with input laser power with a slope of $15 \mathrm{pm}$ per $\mathrm{mW}$. Thus, a $10 \mathrm{~mW}$ laser input will produce a $150 \mathrm{pm}$ reflection peak shift. This is in contrast to a $20 \mathrm{pm}$ shift in water and a $5 \mathrm{pm}$ shift in liquid nitrogen. The grating wavelength shifts in water and liquid $\mathrm{N}_{2}$ were re-plotted using a reduced vertical scale as shown in the inset of the same figure. Compared with air, most liquids have much larger specific heat capacities and thermal convection rates, therefore, it is much harder to raise the temperature of the grating in liquid than in an air/gas environment. Furthermore, when the grating temperature rises up to the boiling point of the surrounding liquid ( $77 \mathrm{~K}$ for liquid $\mathrm{N}_{2}$ ), the temperature of the grating will be held at the boiling point until the surrounding liquid is totally evaporated. This is clearly illustrated in the

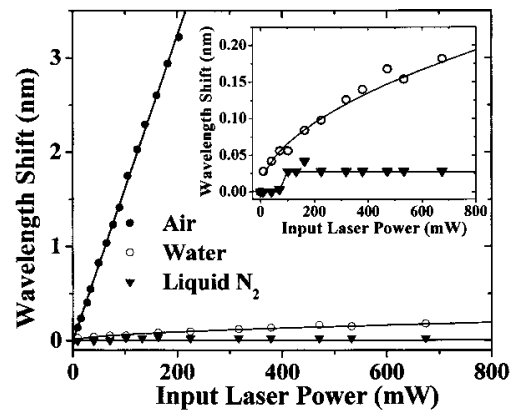

FIG. 4. Resonance wavelength shifts of heated gratings in air, water, and liquid $\mathrm{N}_{2}$ as a function of input laser power. The grating wavelength shifts in water and liquid $\mathrm{N}_{2}$ re-plotted using a reduced vertical scale is shown in the inset of the same figure. 


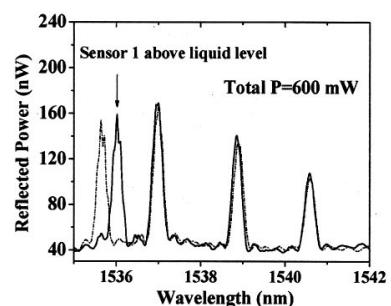

(a)

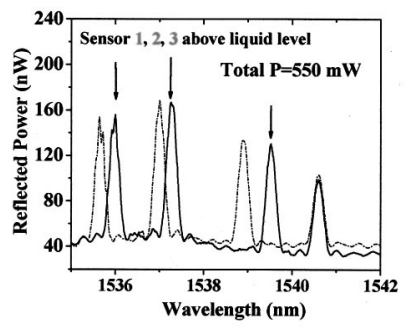

(c)

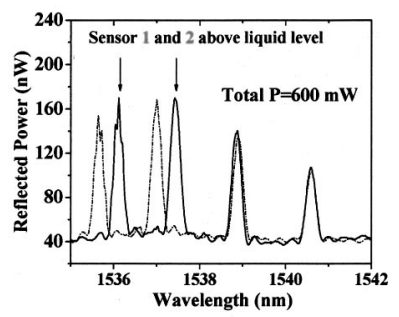

(b)

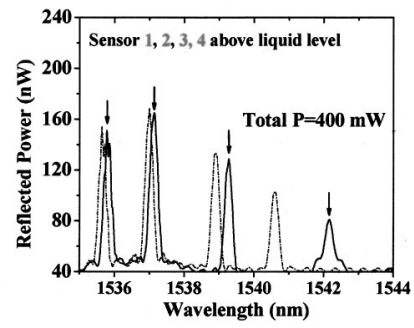

(d)
FIG. 5. Spectra responses of a four-grating sensor array when the fiber was pulled out in serial from water. Sensor 1 was the topmost grating and Sensor 4 was the lowest sensor. The dot traces are the spectral responses for the unheated sensor.

inset of Fig. 4. The resonance wavelength of FBG increased only by $20 \mathrm{pm}$ and subsequently remained constant over varying injection power up to $680 \mathrm{~mW}$. In contrast, the reflection peak of the FBG submerged in water increased monotonically to $170 \mathrm{pm}$ with the same injected laser power.

Based on this experimental setup, a four-point sensor array was also demonstrated to test the liquid/gas discrimination by raising the sensor array from the liquid. To demonstrate a sensor array, the $100 / 400 \mu \mathrm{m}$ multimode fiber was replaced by a $62.5 \mu \mathrm{m}$ core $/ 125 \mu \mathrm{m}$ cladding graded index multimode fiber to increase the power laser light propagation distance from the splice junction. This fiber provided better mode matching than the previous multimode fiber. The acrylic coating of the single-mode fiber was carefully stripped to ensure a clean silica fiber surface.

An array of four $5 \mathrm{~mm}$ gratings was written in a singlemode fiber (Corning SMF-28). The grating sensors were 3 $\mathrm{cm}$ apart with resonance wavelengths at $1535.6 \mathrm{~nm}$ (sensor 1), $1537 \mathrm{~nm}$ (sensor 2), $1538.8 \mathrm{~nm}$ (sensor 3), and $1540.6 \mathrm{~nm}$ (sensor 4), respectively. Sensor 4 was approximately $2 \mathrm{~cm}$ from the splice junction. The reflection spectrum of the unheated grating array is shown as the dot trace in Fig. 5. Sensor 1 (with the shortest resonance wavelength) was the topmost grating, and Sensor 4 (with the longest resonance wavelength) was the lowest grating. Figure 5(a) shows the reflection spectrum of the optically heated grating array when Sensor 1 (topmost) is pulled out from the water with $600 \mathrm{~mW}$ input laser power. The resonance peak for Sensor 1 shifted $350 \mathrm{pm}$ from 1535.7 to $1536.05 \mathrm{~nm}$. Due to the much larger specific heat and thermal convection rates of water than those of air, reflection peaks for heated gratings remaining in the water shifted less than $10 \mathrm{pm}$. In practice, a smaller wavelength shift would be enough to ensure unambiguous detection of the liquid level. Similar behavior was observed when Sensors 2, 3 and 4 were pulled out from water in serial. Figures 5(c) and 5(d) also show nonuniform peak shifts for the different gratings while the input laser power is reduced to $550 \mathrm{~mW}$ and then to $400 \mathrm{~mW}$. This is due to the nonuniform leakage profile of the laser light in a single-mode fiber. The power was reduced to avoid the spectral deformation of Sensor 4 due to overheating.

In conclusion, this work illustrates an innovative solution to power in-fiber components and to enhance the functionality of passive fiber sensor networks. We use fibers not only for optical signal delivery, but also as multi-function cables that can deliver optical power for on-fiber selfheating. The concept of active fiber sensors directly driven by in-fiber light provides a dimension to design agile and multifunctional fiber sensors without sacrificing any intrinsic advantages of passive fiber sensors. In this letter, an optically heated fiber Bragg grating sensor was used to unambiguously determine the liquid level at room temperature as well as at cryogenic temperatures. It promises an ideal solution for cryogenic fuel gauging in a microgravity environment. The optical energy utilized herein can also be converted into energy of other forms for on-fiber actuation. We believe that the ability of tuning in-fiber devices directly by in-fiber optical energy will enable a wide array of agile fiber devices based on FBGs, fiber interferometers, and microstructure fibers. On the other hand, the optical power supply configuration presented in this letter still requires two optical feedthrough lines to inject sensing light and power light from the opposite side of fiber. This drawback can be overcome by using a double-clad fiber for both optical power and sensing signal delivery. Using double-clad specialty fibers, the optical power source can be launched together with the $1.55 \mu \mathrm{m}$ probing light source from the same side to power a large grating array. Optical taps created by ion implantation can release the optical power only at sections of the fiber where FBGs are located. The entire fiber network will only use one feedthrough to connect the detection/power unit to the fiber network. The demonstration of light-powered tunable grating arrays using double-clad fiber will be presented elsewhere.

${ }^{1}$ K. H. Hill, Y. Fujii, D. C. Johnson, and B. S. Kawasaki, Appl. Phys. Lett. 32, 647 (1978).

${ }^{2}$ J. A. Rogers, B. J. Eggleton, J. Pedrazzani, and T. Strasser, Appl. Phys. Lett. 74, 3131 (1999).

${ }^{3}$ H. G. Limberger, N. H. Ky, D. M. Costantini, R. P. Salathe, C. A. P. Muller, and G. R. Fox, IEEE Photonics Technol. Lett. 10, 361 (1998).

${ }^{4}$ P. Mach, M. Dolinski, K. W. Baldwin, J. A. Rogers, C. Kerbage, R. S. Windeler, and B. J. Eggleton, Appl. Phys. Lett. 80, 4294 (2002).

${ }^{5}$ P. Mach, T. Krupenkin, S. Yang, and J. A. Rogers, Appl. Phys. Lett. 81, 202 (2002).

${ }^{6}$ G. R. Fox, C. A. P. Muller, N. Setter, D. M. Costantini, N. H. Ky, and H. G. Limberger, J. Vac. Sci. Technol. A 15, 1791 (1994).

${ }^{7}$ C. Goh, M. Mokhtar, S. Bulter, S. Set, K. Kikuchi, and M. Ibsen, IEEE Photonics Technol. Lett. 15, 557 (2003).

${ }^{8}$ K. P. Chen, L. J. Cashdollars, and W. Xu, IEEE Photonics Technol. Lett. 16, 1897 (2003). 